\title{
Association between Consumption of Sugar-Sweetened Beverages and Risk of Cardiovascular Disease in Korean Men: Analysis Based on the Korea National Health and Nutrition Examination Survey 2014-2016
}

\author{
Su-Ok Kim¹, Eun Mi Bae'², Ye-Na Lee', Joung Sik Son ${ }^{3, *}$ \\ 'Department of Family Medicine, Seoul National University Hospital, Seoul, Korea \\ ${ }^{2}$ Department of Family Medicine, National Police Hospital, Seoul, Korea \\ ${ }^{3}$ Department of Family Medicine, Korea University Guro Hospital, Seoul, Korea
}

Background: This study aimed to evaluate the association between the high risk of cardiovascular disease (CVD) and the consumption of sugar-sweetened beverages (SSBs) in Korean men.

Methods: This cross-sectional study analyzed the data of 3,705 men (age 30-64 years) who participated in the 2014-2016 Korea National Health and Nutrition Examination Survey (KNHANES). SSB intake was defined as the sum of the intakes of carbonated beverages and fruit juices. Participants were categorized into study groups depending on their intake of SSBs: $\leq 2,3-4$, or $\geq 5$ times per week. High CVD risk was defined as a 10-year risk of more than 10\%, based on the Framingham Heart Study 10-year CVD Risk Calculator. The association between high CVD risk and SSB intake was evaluated using a multivariable-adjusted logistic regression model.

Results: Korean men who consumed SSBs 3-4 and $\geq 5$ times a week showed a multivariate-adjusted odds ratio of 1.49 (95\% confidence interval [CI], 1.05-2.11) and 1.61 (95\% CI, 0.97-2.67) for high CVD risk, respectively, compared with those who consumed SSBs $\leq 2$ times per week. Additionally, the risk of CVD increased with the increase in the intake of SSBs (P-trend=0.01). In subgroup analysis, no association was observed between SSB intake and high CVD risk in the group with regular physical activity ( $\mathrm{P}$ for interaction $=0.01$ ).

Conclusion: In Korean men, except those with regular physical activity, SSB intake $\geq 3$ times a week is associated with a high risk for CVD (10-year CVD risk $\geq 10 \%)$.

Keywords: Sugar-Sweetened Beverages; Cardiovascular Diseases; Korean; Men

Received: May 15, 2020, Revised: September 17, 2020, Accepted: September 23, 2020

${ }^{*}$ Corresponding Author: Joung-Sik Son https://orcid.org/0000-0003-2460-3378

Tel: +82-2-2626-1114, Fax: +82-2-2626-2024, E-mail: medical114@naver.com 


\section{INTRODUCTION}

In Korea, aging of the population has resulted in a progressive increase in the healthcare burden conferred by chronic diseases such as cancer, cardiovascular disease (CVD), obesity, diabetes, and osteoporosis. In particular, CVD follows malignant neoplasms as the second leading cause of death in Korea. Therefore, decreasing the incidence and prevalence of CVD has become an important public health goal to improve population health and reduce medical expenses. The World Health Organization has indicated a high correlation between the dietary intake and the occurrence of chronic diseases. ${ }^{1)}$ Thus, dietary improvements are very important, as dietary factors have a higher contribution than do smoking and drinking to the burden of chronic diseases. ${ }^{2}$ Based on their findings from a 34-year, large-scale prospective cohort study, Malik et al. ${ }^{3)}$ reported that the ingestion of sugar-sweetened beverages (SSBs) was associated with CVD, and the resultant mortality directly increased with the increased intake of SSBs. Several studies have reported an increased risk of chronic diseases, such as obesity, type 2 diabetes, hypertension, and metabolic syndrome, with the increased intake of SSBs. ${ }^{4,5)}$ Processed foods account for a large portion of the Korean diet at present, ${ }^{6)}$ and more than $74 \%$ of processed foods contain added sugars. ${ }^{7)}$ Beverages account for the highest proportion $(34.3 \%)$ of sugar intake from processed foods, ${ }^{8)}$ with beverages that contain liquid sugar syrup constituting the majority. ${ }^{9)}$ In Korea, the average intake of SSBs was 299.2 and $208.8 \mathrm{~g}$ /day for men and women, respectively, in 2013-2015. This indicated an increase of 59.6\% and $76.8 \%$ in intake for men and women, respectively, compared with the intakes in 2007-2009. ${ }^{10)}$ Nonetheless, the association between the high risk of CVD and the consumption of SSBs has not been adequately evaluated in Korean men.

This study was undertaken to confirm the association between the intake of SSBs and the high risk of CVD in Korean men by using the data from the 2014-2016 Korean National Health and Nutrition Examination Survey (KNHANES), and in so doing, provide the requisite evi- dence for health policy changes with the goal of CVD prevention.

\section{METHODS}

\section{Participants}

This cross-sectional study used data from the KNHANES, which was conducted in 2014-2016 and included health surveys, screening, and food intake-frequency surveys. Data collected from adult males (age 30-64 years) were obtained for analysis. A total of 4,882 people were surveyed in the 2014-2016 KNHANES. The data of individuals who (1) had been diagnosed with or treated for CVD, cancer, or other chronic diseases (chronic renal failure, cirrhosis), and/or (2) had a total energy intake of less than $800 \mathrm{kcal}$ per day or more than 5,000 kcal per adult male, and/or (3) had not responded to the food intake-frequency surveys of SSBs (carbonated drinks and fruit juices) were excluded from the study. The final analysis dataset of this study included the data of 3,705 individuals (Figure 1). This study was approved by the Medical Research Ethics Review Committee of the Seoul National University Hospital (IRB approval no., E-2002-001-1097). The requirement for informed consent from individual patients was omitted because of the retrospective design of this study.

\section{Study Variables}

Sociodemographic variables such as age, marital status, education level, employment status, and level of household income were surveyed. The education level was categorized into elementary school or less, middle school, high school, and college or higher education. The employment status was classified as employed and unemployed. The household income levels were categorized as low, middle-low, middle-high, and high in accordance with the income quartile (household). Body mass index (BMI) was calculated by dividing the individual's weight $(\mathrm{kg})$ by the square of his height $(\mathrm{m})$. Health-related behaviors such as smoking, drinking, and regular physical activity were ascertained through the questionnaire survey. Based on the smoking 
status, participants were categorized as never smokers, ex-smokers, and current smokers. Never smokers had never smoked or smoked less than five packs ( $\leq 100$ cigarettes) in their lifetime and were not currently smoking; ex-smokers had smoked more than five packs of cigarettes in their lifetime but had quit smoking; and current smokers had smoked more than five packs of cigarettes in their lifetime, and currently smoked. Drinking was defined as the consumption of more than one drink per month in the past year, based on the monthly drinking rate. Regular physical activity was defined as $\geq 2.5$ hours of moderateintensity physical activity, $\geq 1.25$ hours of high-intensity physical activity, or a combination of both ( 1 minute of high-intensity activity is equivalent to 2 minutes of moderate-intensity activity) per week. Chronic diseases such as hypertension, diabetes, and dyslipidemia were considered only on the basis of diagnosis or treatment by a doctor. With regard to mental health, stress status was defined as high or very high levels of stress based on the self-perception rate of stress. The daily intake of energy, carbohydrates, proteins, and fats was calculated through the food intake-frequency survey.

\section{Definition of Sugar-Sweetened Beverages}

In 2017, the Global Burden of Disease Risk Factor Collaborators included carbonated drinks, sodas, energy drinks, and fruit juices as SSBs, ${ }^{11)}$ and excluded juices that were made entirely from fruits or vegetables from the SSB group. In the food intake-frequency survey, coke, cider, and carbonated fruit beverages were included as carbonated drinks, and the average intake of carbonated beverages and fruit juices for the past 1 year was surveyed. Most fruit juices contain sugar syrup and can, thus, be considered as SSBs. Therefore, in this study, the intake of SSBs was defined as the sum of the intakes of carbonated beverages (colas, cider, and carbonated fruit beverages) and fruit juices. The SSBs intake groups were further subcategorized as groups that consumed SSBs $\leq 2$ times a week, 3-4 times a week, and $\geq 5$ times a

Table 1. General characteristics of study participants based on the consumption of SSBS

\begin{tabular}{|c|c|c|c|c|c|}
\hline \multirow{2}{*}{ Characteristic } & \multicolumn{4}{|c|}{ Consumption of SSB (times/wk) } & \multirow{2}{*}{ P-value } \\
\hline & $\leq 2$ & $3-4$ & $\geq 5$ & Total & \\
\hline No. of participants & $2,440(65.7)$ & $410(11.1)$ & $239(6.5)$ & 3,705 & \\
\hline Age (y) & $46.5 \pm 0.3$ & $40.8 \pm 0.4$ & $40 \pm 0.6$ & $45.5 \pm 0.2$ & $<0.01$ \\
\hline Married & $1,984(86.9)$ & $342(82.8)$ & $410(75.1)$ & 2,914 (85.4) & $<0.01$ \\
\hline \multicolumn{6}{|l|}{ Education } \\
\hline Elementary school or less & $129(5.7)$ & $13(3.1)$ & $9(3.7)$ & $200(5.9)$ & $<0.01$ \\
\hline Middle school & $158(6.9)$ & $21(5.1)$ & $7(2.8)$ & $231(6.8)$ & \\
\hline High school & 667 (29.2) & $101(24.5)$ & $61(25.9)$ & $983(28.8)$ & \\
\hline College or more & $1,123(49.2)$ & $231(55.8)$ & $132(55.6)$ & $1,651(48.4)$ & \\
\hline Employed & $1,877(82.2)$ & $335(81.1)$ & $192(91.0)$ & $2,759(80.9)$ & $<0.01$ \\
\hline \multicolumn{6}{|l|}{ Household income } \\
\hline Low & $147(6.5)$ & $15(3.7)$ & $12(4.9)$ & $241(7.0)$ & $<0.01$ \\
\hline Middle-low & $501(22.0)$ & $89(21.4)$ & $51(21.6)$ & $766(22.5)$ & \\
\hline Middle-high & $741(32.5)$ & $148(35.9)$ & $89(37.4)$ & $1,097(32.2)$ & \\
\hline High & $883(38.7)$ & $161(38.9)$ & $86(36.1)$ & $1,292(37.9)$ & \\
\hline Body mass index (kg/m²) & $24.8 \pm 0.1$ & $24.6 \pm 0.2$ & $25.2 \pm 0.3$ & $24.8 \pm 0.1$ & 0.21 \\
\hline \multicolumn{6}{|l|}{ Smoking } \\
\hline Never smoker & $532(23.3)$ & $94(22.7)$ & $47(19.9)$ & 757 (22.2) & $<0.01$ \\
\hline Ex-smoker & $754(33.1)$ & $121(29.2)$ & $62(26.1)$ & $1,081(31.7)$ & \\
\hline Current smoker & 987 (39.3) & $179(43.5)$ & $115(48.4)$ & $1,415(41.5)$ & \\
\hline Alcohol use ${ }^{*}$ & $1,673(73.3)$ & $302(73.1)$ & $178(74.7)$ & 2,495 (73.2) & $<0.01$ \\
\hline Regular $\mathrm{PA}^{\dagger}$ & $1,110(48.6)$ & $196(47.4)$ & $119(49.9)$ & $1,620(47.5)$ & $<0.01$ \\
\hline Hypertension ${ }^{\ddagger}$ & $329(14.4)$ & $37(9.0)$ & $12(5.1)$ & $469(13.7)$ & $<0.01$ \\
\hline Diabetes mellitus ${ }^{\ddagger}$ & $140(6.1)$ & $13(3.2)$ & $3(1.0)$ & $188(5.5)$ & $<0.01$ \\
\hline Dyslipidemia $^{\ddagger}$ & $253(11.1)$ & $35(8.4)$ & $19(8.1)$ & $354(10.4)$ & $<0.01$ \\
\hline Stressed ${ }^{\S}$ & $585(25.7)$ & $123(29.6)$ & 91 (38.2) & $934(27.4)$ & $<0.01$ \\
\hline Total energy intake (kcal/d) & $2,191 \pm 17.0$ & $2,516 \pm 41.6$ & $2,785 \pm 63.9$ & $2,285 \pm 15.9$ & $<0.01$ \\
\hline Carbohydrate intake (g/d) & $333 \pm 2.9$ & $373 \pm 5.7$ & $411 \pm 9.0$ & $346 \pm 2.1$ & $<0.01$ \\
\hline Protein intake $(\mathrm{g} / \mathrm{d})$ & $69 \pm 0.7$ & $83 \pm 1.7$ & $92 \pm 3.0$ & $73 \pm 0.7$ & $<0.01$ \\
\hline Total fat intake (g/d) & $41 \pm 0.6$ & $54 \pm 1.3$ & $61 \pm 2.2$ & $44 \pm 0.5$ & $<0.01$ \\
\hline
\end{tabular}

Values are presented as number (\%) or mean \pm standard error. P-values were calculated using the chi-square test or one-way analysis of variance. P $<0.05$ indicate statistical significance.

SSBs, sugar-sweetened beverages; PA, physical activity.

${ }^{*}$ Alcohol use is defined as consumption of one or more glasses of alcohol per month in the past year. 'Regular PA was defined as $\geq 2.5$ hours of moderate-intensity PA, $\geq 1.25$ hours of high-intensity PA, or a combination of both (1 minute of high-intensity activity is equivalent to 2 minutes of moderate-intensity activity) per week. ${ }^{*}$ Hypertension, diabetes mellitus, and dyslipidemia are based on diagnosis by physicians or receiving treatment for each disease. ${ }^{\S}$ Those under considerable stress, based on the selfperception rate of stress, are defined as stressed. 
week.

\section{Definition of High Risk of Cardiovascular Disease}

The risk of CVD was calculated using the Framingham Heart Study 10year CVD Risk Calculator. Indicators such as age, gender, systolic blood pressure, hypertension treatment, smoking, diabetes, high-density lipoprotein cholesterol, and total cholesterol were used for risk calculation. A 10 -year CVD risk of $10 \%$ or higher was defined as a high risk of CVD.

\section{Statistical Analysis}

In accordance with the characteristics of the KNHANES, which has a complex sampling design, this study conducted analyses by the weighting, stratification, and clustering of variables. Age; BMI; total daily energy intake; and daily carbohydrate, protein, and fat intake were all calculated as the mean and standard error. One-way analysis of variance was used to verify the differences between the groups based on the intake of SSBs. Marital status, education level, employment status, household income level, smoking, drinking, regular physical activity, hypertension, diabetes, dyslipidemia, and stress were all calculated frequencies and percentages (\%). The chi-square test was used to verify the inter-group differences based on the intake of SSBs. To evaluate the association between the intake of SSBs and high risk of CVD, a multivariable-adjusted logistic regression analysis was conducted for each group stratified by SSB intake. After adjusting the variables in the three models developed for this study, the odds ratios (OR) for each SSB intake group were calculated. All analyses were conducted using Stata/MP ver. 15.0 (Stata Corp., College Station, TX, USA) and were interpreted as significant only when the P-value was less than 0.05 .

\section{RESULTS}

\section{General Characteristics of the Study Population}

Table 1 shows the general characteristics of the study participants based on the intake of SSBs. The intake frequency of SSBs ranged from 0 to 28 times per week. We found that 2,440 (65.7\%), 410 (11.1\%), and 239 (6.5\%) participants consumed SSBs $\leq 2,3-4$, and $\geq 5$ times a week, respectively. The average age of participants in this study population was 45.5 years, whereas the average age in the groups that consumed SSBs $\leq 2$ and $\geq 5$ times per week was 46.5 and 40 years, respectively. Our results show that the intake of SSBs increased inversely with reference to the average age. The proportion of married individuals was lowest (75.1\%) in the group that consumed SSBs $\geq 5$ times a week. The average BMI was highest, at $25.2 \mathrm{~kg} / \mathrm{m}^{2}$, in the group that consumed SSBs $\geq 5$ times a week, but there was no significant difference in the BMIs of the three groups. The current smoking rate was the highest (48.4\%) in the group that consumed SSBs $\geq 5$ times a week. In the group that had a high proportion of participants with hypertension, diabetes, and dyslipidemia, the intake of SSBs was low. The stress rate was highest (38.2\%) in the group that consumed SSBs $\geq 5$ times a week. The intake of total energy, carbohydrates, proteins, and fats intakes per day was highest in the group that consumed SSBs $\geq 5$ times a week.

\section{Association between the Intake of Sugar-Sweetened Beverages and the High Risk of Cardiovascular Disease}

Table 2 shows the OR and the 95\% confidence intervals (95\% CI) for the high risk of CVD based on the consumption of SSBs per week. In the age-adjusted model 1, compared to the group that consumed SSBs $\leq 2$ times a week, the OR for groups that consumed SSBs 3-4 and $\geq 5$ times a week was 1.32 (95\% CI, 0.95-1.83), and 1.53 (95\% CI, 0.94$2.48)$, respectively. These differences were not significant. However, as the intake of SSBs increased, the risk for CVD increased (Ptrend=0.03). In model 2 , in addition to model 1 , we adjusted for marital status, education level, employment status, household income level, BMI, drinking, regular physical activity, dyslipidemia, and stress variables. Compared to the group that consumed SSBs $\leq 2$ times a week, the ORs for a high risk of CVD in groups that consumed SSBs 3-4 and $\geq 5$ times a week were 1.41 (95\% CI, 1.01-1.98) and 1.54 (95\% CI, 0.94-2.51), respectively. The risk of CVD increased with the increase in the intake of SSBs (P-trend=0.02). In model 3, in addition to model 2, we adjusted the total daily intake of carbohydrates, proteins, and fats. In comparison with the group that consumed SSBs $\leq 2$ times a week, the OR for high risk of CVD in the groups that consumed SSBs 3-4 and $\geq 5$ times a week was 1.49 (95\% CI, 1.05-2.11) and 1.61 (95\% CI, 0.972.67), respectively. As in models 1 and 2, the risk of CVD increased with the increase in the intake of SSBs (P-trend=0.01). Table 3 shows the OR and $95 \%$ CI for high risk of CVD on the basis of SSB intake per

Table 2. ORs and $95 \%$ Cls for the high risk of CVD based on the consumption of SSBs

\begin{tabular}{|c|c|c|c|c|}
\hline \multirow{2}{*}{ Variable } & \multicolumn{3}{|c|}{ Consumption of SSB (times/wk) } & \multirow{2}{*}{$\mathrm{P}$ trend } \\
\hline & $\leq 2$ & $3-4$ & $\geq 5$ & \\
\hline Model 1 & 1.00 & $1.32(0.95-1.83)$ & $1.53(0.94-2.48)$ & 0.03 \\
\hline Model 2 & 1.00 & $1.41(1.01-1.98)$ & $1.54(0.94-2.51)$ & 0.02 \\
\hline Model 3 & 1.00 & $1.49(1.05-2.11)$ & $1.61(0.97-2.67)$ & 0.01 \\
\hline
\end{tabular}

Values are presented as odds ratio (95\% confidence interval). High CVD risk is defined as has a 10-year CVD risk $\geq 10 \%$. ORs and P trends were estimated using multivariateadjusted logistic regression analysis. Model 1: adjusted for age; model 2: adjusted for model 1 plus marital status, education, employment, household income, body mass index, alcohol use, regular physical activity, dyslipidemia, and stress; and model 3: adjusted for model 2 plus carbohydrate intake (g/d), protein intake (g/d), and total fat intake $(\mathrm{g} / \mathrm{d})$.

OR, odds ratio; Cl, confidence interval; CVD, cardiovascular disease; SSBs, sugar-sweetened beverages. 
Table 3. ORs and 95\% Cls for the high risk of CVD based on the consumption of SSBs in the subgroups

\begin{tabular}{|c|c|c|c|c|c|}
\hline \multirow{2}{*}{ Variable } & \multicolumn{3}{|c|}{ Consumption of SSB (times/wk) } & \multirow{2}{*}{$\mathrm{P}$ trend } & \multirow{2}{*}{ P for interaction } \\
\hline & $\leq 2$ & $3-4$ & $\geq 5$ & & \\
\hline Age (y) & & & & & 0.21 \\
\hline$<45$ & 1.00 & $0.90(0.47-1.75)$ & $2.39(1.17-4.88)$ & $<0.05$ & \\
\hline$\geq 45$ & 1.00 & $1.31(0.82-2.10)$ & $0.78(0.39-1.56)$ & 0.93 & \\
\hline BMl $\left(\mathrm{kg} / \mathrm{m}^{2}\right)$ & & & & & 0.18 \\
\hline$<25$ & 1.00 & $1.43(0.84-2.46)$ & $1.33(0.47-3.77)$ & 0.34 & \\
\hline$\geq 25$ & 1.00 & $1.45(0.86-2.43)$ & $1.84(0.94-3.61)$ & 0.04 & \\
\hline \multicolumn{6}{|l|}{ Regular PA* } \\
\hline Yes & 1.00 & $1.45(0.84-2.52)$ & $1.29(0.60-2.78)$ & 0.27 & 0.01 \\
\hline No & 1.00 & $1.60(0.99-2.60)$ & $2.35(1.08-5.10)$ & 0.01 & \\
\hline \multicolumn{6}{|c|}{ Total energy intake (kcal/d) } \\
\hline$<2,400$ & 1.00 & $1.48(0.87-2.51)$ & $1.25(0.54-2.90)$ & 0.26 & 0.23 \\
\hline$\geq 2,400$ & 1.00 & $1.59(0.95-2.68)$ & $2.01(1.01-4.00)$ & 0.02 & \\
\hline \multicolumn{6}{|c|}{ Carbohydrate intake (g/d) } \\
\hline$<350$ & 1.00 & $1.63(0.95-2.78)$ & $1.37(0.58-3.24)$ & 0.16 & 0.06 \\
\hline$\geq 350$ & 1.00 & $1.33(0.81-2.19)$ & $1.66(0.83-3.31)$ & 0.10 & \\
\hline
\end{tabular}

Values are presented as odds ratio (95\% confidence interval). High CVD risk is defined as has a 10-year CVD risk $\geq 10 \%$. ORs and P trends for age in subgroup were estimated using multivariate logistic regression analysis after adjusting for marital status, education, employment, household income, BMl, alcohol use, regular PA, dyslipidemia, stress, carbohydrate intake $(\mathrm{g} / \mathrm{d})$, protein intake $(\mathrm{g} / \mathrm{d})$, and total fat intake $(\mathrm{g} / \mathrm{d})$. ORs and $\mathrm{P}$ trends for BMl in subgroup were estimated using multivariate logistic regression analysis after adjusting for age marital status, education, employment, household income, alcohol use, regular PA, dyslipidemia, stress, carbohydrate intake ( $\mathrm{g} / \mathrm{d})$, protein intake ( $\mathrm{g} / \mathrm{d})$, and total fat intake $(\mathrm{g} / \mathrm{d})$. ORs and P trends for regular PA in subgroup were estimated using multivariate logistic regression analysis after adjusting for age marital status, education, employment, household income, BMl, alcohol use, dyslipidemia, stress, carbohydrate intake $(\mathrm{g} / \mathrm{d})$, protein intake $(\mathrm{g} / \mathrm{d})$, and total fat intake $(\mathrm{g} / \mathrm{d})$. ORs and P trends for total energy intake in subgroup were estimated using multivariate logistic regression analysis after adjusting for age marital status, education, employment, household income, BMI, alcohol use, regular PA, dyslipidemia, stress, carbohydrate intake $(\mathrm{g} / \mathrm{d})$, protein intake $(\mathrm{g} / \mathrm{d})$, and total fat intake $(\mathrm{g} / \mathrm{d})$. ORs and P trends for carbohydrate intake in subgroup were estimated using multivariate logistic regression analysis after adjusting for age marital status, education, employment, household income, BMl, alcohol use, regular PA, dyslipidemia, stress, protein intake $(\mathrm{g} / \mathrm{d})$, and total fat intake $(\mathrm{g} / \mathrm{d})$.

OR, odds ratio; $\mathrm{Cl}$, confidence interval; CVD, cardiovascular disease; SSBs, sugar-sweetened beverages; BMI, body mass index; PA, physical activity.

${ }^{*}$ Regular PA was defined as $\geq 2.5$ hours of moderate-intensity PA, $\geq 1.25$ hours of high-intensity PA, or a combination of both (1 minute of high-intensity activity is equivalent to 2 minutes of moderate-intensity activity) per week.

week by the different subgroups. Among participants younger than 45 years (wherein age $>45$ years is one of the risk factors for CVD in men), the risk of CVD was higher in the group that consumed SSBs $\geq 5$ times a week (OR, 2.39; 95\% CI, 1.17-4.88) than in the group that consumed SSBs $\leq 2$ times a week. The risk of CVD increased with the increased intake of SSBs (P-trend <0.05). However, this group showed no significant difference from the group over the age of 45 years ( $P$ for interaction=0.21). Among participants with BMI $\geq 25 \mathrm{~kg} / \mathrm{m}^{2}$ (obesity criterion), the OR for high risk of CVD in groups that consumed beverages 3-4 and $\geq 5$ times a week was 1.45 (95\% CI, 0.86-2.43) and 1.84 (95\% CI, 0.94-3.61), respectively, compared to in the group that consumed SSBs $\leq 2$ times a week. The risk of CVD increased as the intake of SSBs increased (P-trend=0.04). However, this group showed no difference from the group with $\mathrm{BMI}<25 \mathrm{~kg} / \mathrm{m}^{2}$ (P for interaction=0.18). Among participants without regular physical activity, the ORs for high risk of CVD in groups that consumed SSBs 3-4 and $\geq 5$ times a week were 1.60 (95\% CI, 0.99-2.60) and 2.35 (95\% CI, 1.08-5.10), respectively, compared to that in the group that consumed SSBs $\leq 2$ times a week. The risk of CVD increased with the increase in the intake of SSBs (Ptrend=0.01). However, in the group with regular physical activity, no association was observed between SSB intake and high CVD risk ( $\mathrm{P}$ for interaction=0.01). Among participants who consumed $\geq 2,400 \mathrm{kcal} / \mathrm{d}$, the ORs for high risk of CVD in groups that consumed SSBs $3-4$ and $\geq 5$ times a week were 1.59 (95\% CI, 0.95-2.68) and 2.01 (95\% CI, 1.01-
$4.00)$, respectively, compared to those in the group that consumed SSBs $\leq 2$ times a week. The risk of CVD increased with the increased intake of SSBs (P-trend=0.02). However, this group showed no difference from the group that consumed $<2,400 \mathrm{kcal} / \mathrm{d}$ ( $\mathrm{P}$ for interaction=0.23).

\section{DISCUSSION}

This study evaluated the association between the intake of SSBs and the high risk of CVD in Korean men using the data from the 2014-2016 KNHANES. The present study demonstrated a significant association between the consumption of SSBs and the high risk of CVD, especially in individuals who consume these beverages $\geq 3$ times a week. These findings are consistent with those of previous studies, which showed that the consumption of SSBs was related to a high risk of CVD. ${ }^{12-15)}$ In a meta-analysis of four prospective studies, Huang et al. ${ }^{16)}$ showed that the relative risk for coronary heart disease (CHD) increases with the increased intake of SSBs. With a once-daily increase in the intake of SSBs, the relative risk of CHD increased by $16 \%$, and this association was reported to be particularly pronounced in men. We found that, in Korean men, the OR for high risk of CVD increased by $49 \%$ with the consumption of SSBs 3-4 times a week compared with those who consumed SSBs $\leq 2$ times per week. Malik and $\mathrm{Hu}^{17}$ ) reported that SSBs have a strong positive association with weight gain, type 2 diabetes, 
and CHD risk, regardless of the fat intake. The relative risk of type $2 \mathrm{di}-$ abetes was reported to be 1.28 (95\% CI, 1.12-1.46) with SSBs, 1.29 (95\% CI, 1.08-1.54) with artificially sweetened beverages (ASBs), and 1.10 (95\% CI, 1.01-1.20) with fruit juices. Kim et al. ${ }^{18)}$ conducted a study using the data from the US National Health and Nutrition Examination Survey, and reported an increased risk of high blood pressure (87\%; $95 \%$ CI, 1.06-3.26), which is one of the risk factors for CVD, in individuals who consume SSBs $\geq 3$ times a day compared to participants who did not. Several mechanisms have been suggested whereby SSBs can cause CVD. Malik and $\mathrm{Hu}^{19)}$ and Stanhope ${ }^{20)}$ have suggested that SSBs provide less satiety than actual calories. This indicates that the consumption of SSBs does not result in a decreased food intake; instead, this consumption can lead to a rapid increase in blood sugar that can promote an increase in appetite and result in overall weight gain. In addition, fructose-containing sugars can promote gout and dyslipidemia by promoting the production of uric acid, triglycerides, and cholesterol in the liver. Nakagawa et al. ${ }^{21)}$ have shown that increased blood levels of uric acid caused by the increased fructose intake decreases the levels of nitric oxide, which, in turn, impairs the functions of the vascular endothelium and increases the risk of CVD. Furthermore, SSBs can promote insulin secretion, with resultant retention of sodium and water in the body, which subsequently cause an increase in blood pressure. ${ }^{22)}$ Some studies have reported that replacing SSBs with ASBs may reduce the risk of $\mathrm{CHD}^{23)}$ However, Imamura et al. ${ }^{24)}$ and Malik et al. ${ }^{3)}$ have reported that both SSBs and ASBs increase type 2 diabetesand CVD-associated mortality rates. Based on a computer-simulated study, Mekonnen et al. ${ }^{25)}$ predicted that reducing the intake of SSBs by $10 \%-20 \%$ over the next 10 years will have significant health benefits and cost savings. In another study that reviewed the epidemiological and biochemical evidence, Thornley et al. ${ }^{26)}$ recommended that clinicians who undertake CVD counseling should advise patients to limit their sugar intake. Therefore, the steadily increasing intake of SSBs is an important factor in the increased prevalence and mortality of CVD, which makes it imperative to reduce SSB intake to ensure population health. In the past 30 years, most studies have focused on the role of saturated fats and sodium in CVDs. However, more recent studies have evaluated the relationship between excessive sugar intake and CVD. To confirm the role of sugars in the progression of CVD, there is a need for further research, including prospective studies, in several countries. And in the subgroup analysis, no association was observed between SSB intake and high CVD risk in the group with regular physical activity.

This study has a few limitations. First, given the cross-sectional study design, it was not possible to determine the exact temporal relationship between the intake of SSBs and the high risk of CVD. Second, the intake of SSBs was defined only as the sum of the intakes of carbonated beverages and fruit juices, which can possibly dilute the relationship between SSBs and the high risk of CVD; although carbonated beverages and fruit juices constitute the majority of sweetened beverages. Finally, as the traditional Framingham Risk Score was used, the risk of CVD in Koreans could have been overestimated. ${ }^{27)}$ Nonetheless, the
Framingham Risk Score is one of the most widely applied risk-scoring methods in clinical practice because it can assess the risk of CVD based on easy-to-obtain medical history and cholesterol levels.

In conclusion, our study in Korean men, confirms the findings from previous research studies that a higher intake of SSBs is associated with a higher risk of CVD. The data of the KNHANES used for our analysis were representative of the whole Korean population and allowed an appropriate evaluation of the association while adjusting for several variables. This study found an association between the increased consumption of SSBs and a higher risk of CVD. The findings of this research will provide a basis for measures to improve the dietary intake of the Korean people to reduce the risk for CVD. It is recommended that more accurate data on the intake of added sugars, SSBs, and ASBs among Koreans be obtained through future periodic surveys to enable a more comprehensive evaluation of the risk, incidence, and mortality of CVDs. Prospective cohort studies are also recommended to validate and confirm the findings of this study.

\section{CONFLICT OF INTEREST}

No potential conflict of interest relevant to this article was reported.

\section{ACKNOWLEDGMENTS}

We would like to thank Minsoo Kang, for his contribution to the research study by extracting and analyzing the statistical data in this study.

\section{ORCID}

Su-Ok Kim: https://orcid.org/0000-0001-9514-0695

Eun Mi Bae: https://orcid.org/0000-0002-4565-9090

Ye-Na Lee: https://orcid.org/0000-0002-6356-3121

Joung Sik Son: https://orcid.org/0000-0003-2460-3378

\section{REFERENCES}

1. Kim HR. Nutrition transition and shifting diet linked noncommunicable diseases and policy issues. Health Welf Policy Forum 2013;(198): 27-37.

2. GBD 2015 Risk Factors Collaborators. Global, regional, and national comparative risk assessment of 79 behavioural, environmental and occupational, and metabolic risks or clusters of risks, 1990-2015: a systematic analysis for the Global Burden of Disease Study 2015. Lancet 2016;388:1659-724.

3. Malik VS, Li Y, Pan A, De Koning L, Schernhammer E, Willett WC, et al. Long-term consumption of sugar-sweetened and artificially sweetened beverages and risk of mortality in US adults. Circulation 2019; 139:2113-25.

4. Hwang EJ, Yoo JH, Shin JY, Bae MJ, Jo SI. Association of sugar-sweetened beverages consumption and hypertension in Korean adults: Korean National Health and Nutrition Examination Survey 2012-2013. 
Korean J Fam Pract 2016;6:446-51.

5. Malik VS, Popkin BM, Bray GA, Despres JP, Willett WC, Hu FB. Sugarsweetened beverages and risk of metabolic syndrome and type 2 diabetes: a meta-analysis. Diabetes Care 2010;33:2477-83.

6. Kim SY, Lee JH, Choi JH. Consumers' purchasing patterns and preferences of the processed food products made from domestic ingredients. Korean J Food Mark Econ 2010;27:1-17.

7. Ventura EE, Davis JN, Goran MI. Sugar content of popular sweetened beverages based on objective laboratory analysis: focus on fructose content. Obesity (Silver Spring) 2011;19:868-74.

8. Ministry of Food and Drug Safety. Sugar holic? Korea indulged in sweetness. Cheongju: Ministry of Food and Drug Safety; 2015.

9. Malik AH, Akram Y, Shetty S, Malik SS, Yanchou Njike V. Impact of sugar-sweetened beverages on blood pressure. Am J Cardiol 2014;113: 1574-80.

10. Lim DH, Yoon SH, Oh KW. Trends in dietary risk factors contributing to burden of chronic disease in Korean adults: findings in Korea National Health and Nutrition Examination Survey, 2007-2015. Public Health Wkly Rep 2018;11:27-33.

11. GBD 2017 Risk Factor Collaborators. Global, regional, and national comparative risk assessment of 84 behavioural, environmental and occupational, and metabolic risks or clusters of risks for 195 countries and territories, 1990-2017: a systematic analysis for the Global Burden of Disease Study 2017. Lancet 2018;392:1923-94.

12. Malik VS. Sugar sweetened beverages and cardiometabolic health. Curr Opin Cardiol 2017;32:572-9.

13. Mozaffarian D. Dietary and policy priorities for cardiovascular disease, diabetes, and obesity: a comprehensive review. Circulation 2016;133: 187-225.

14. Micha R, Penalvo JL, Cudhea F, Imamura F, Rehm CD, Mozaffarian D. Association between dietary factors and mortality from heart disease, stroke, and type 2 diabetes in the United States. JAMA 2017;317:91224.

15. Arsenault BJ, Lamarche B, Despres JP. Targeting overconsumption of sugar-sweetened beverages vs. overall poor diet quality for cardiometabolic diseases risk prevention: place your bets! Nutrients 2017;9:600.

16. Huang C, Huang J, Tian Y, Yang X, Gu D. Sugar sweetened beverages consumption and risk of coronary heart disease: a meta-analysis of prospective studies. Atherosclerosis 2014;234:11-6.
17. Malik VS, Hu FB. Sugar-sweetened beverages and cardiometabolic health: an update of the evidence. Nutrients 2019;11:1840.

18. Kim YH, Abris GP, Sung MK, Lee JE. Consumption of sugar-sweetened beverages and blood pressure in the United States: the national health and nutrition examination survey 2003-2006. Clin Nutr Res 2012;1:8593.

19. Malik VS, Hu FB. Fructose and cardiometabolic health: what the evidence from sugar-sweetened beverages tells us. J Am Coll Cardiol 2015;66:1615-24.

20. Stanhope KL. Sugar consumption, metabolic disease and obesity: the state of the controversy. Crit Rev Clin Lab Sci 2016;53:52-67.

21. Nakagawa T, Hu H, Zharikov S, Tuttle KR, Short RA, Glushakova O, et al. A causal role for uric acid in fructose-induced metabolic syndrome. Am J Physiol Renal Physiol 2006;290:F625-31.

22. Fang J, Alderman MH. Serum uric acid and cardiovascular mortality the NHANES I epidemiologic follow-up study, 1971-1992. National Health and Nutrition Examination Survey. JAMA 2000;283:2404-10.

23. Keller A, O'Reilly EJ, Malik V, Buring JE, Andersen I, Steffen L, et al. Substitution of sugar-sweetened beverages for other beverages and the risk of developing coronary heart disease: Results from the Harvard Pooling Project of Diet and Coronary Disease. Prev Med 2020; 131:105970.

24. Imamura F, O'Connor L, Ye Z, Mursu J, Hayashino Y, Bhupathiraju SN, et al. Consumption of sugar sweetened beverages, artificially sweetened beverages, and fruit juice and incidence of type 2 diabetes: systematic review, meta-analysis, and estimation of population attributable fraction. BMJ 2015;351:h3576.

25. Mekonnen TA, Odden MC, Coxson PG, Guzman D, Lightwood J, Wang YC, et al. Health benefits of reducing sugar-sweetened beverage intake in high risk populations of California: results from the cardiovascular disease (CVD) policy model. PLoS One 2013;8:e81723.

26. Thornley S, Tayler R, Sikaris K. Sugar restriction: the evidence for a drug-free intervention to reduce cardiovascular disease risk. Intern Med J 2012;42 Suppl 5:46-58.

27. Hense HW, Schulte H, Lowel H, Assmann G, Keil U. Framingham risk function overestimates risk of coronary heart disease in men and women from Germany: results from the MONICA Augsburg and the PROCAM cohorts. Eur Heart J 2003;24:937-45. 